\title{
Subgroup analysis of efficacy and safety of orantinib in combination with TACE in Japanese HCC patients in a randomized phase III trial (ORIENTAL)
}

\author{
Hisashi Hidaka' (1) $\cdot$ Namiki Izumi $^{2} \cdot$ Takeshi Aramaki $^{3} \cdot$ Masafumi Ikeda $^{4} \cdot$ Yoshitaka Inaba $^{5} \cdot \mathrm{Kazuho} \mathrm{Imanaka}^{6}$. \\ Takuji Okusaka $^{7}$. Susumu Kanazawa ${ }^{8}$. Shuichi Kaneko ${ }^{9}$. Shinichi Kora ${ }^{10}$. Hiroya Saito ${ }^{11}$. Junji Furuse ${ }^{12}$. \\ Osamu Matsui ${ }^{13} \cdot$ Tatsuya Yamashita $^{9}$. Osamu Yokosuka ${ }^{14} \cdot$ Satoshi Morita $^{15} \cdot$ Hitoshi Arioka $^{16} \cdot$ Masatoshi Kudo $^{17}$. \\ Yasuaki Arai $^{18}$
}

Received: 19 February 2019 / Accepted: 17 April 2019 / Published online: 3 May 2019

(c) The Author(s) 2019

\begin{abstract}
A randomized, phase III trial of orantinib in combination with transcatheter arterial chemoembolization (TACE) did not prolong overall survival (OS) over placebo (ORIENTAL study). A subgroup analysis was conducted to evaluate the efficacy and safety of orantinib in Japanese patients enrolled in the ORIENTAL study. The data of Japanese patients from this study were analyzed. The overall survival (OS), time to progression (TTP), and time to TACE failure (TTTF) were compared between orantinib and placebo arms using stratified log-rank test. Since TTTF in patients with Barcelona Clinic Liver Cancer stage B (BCLC-B) showed favor outcome in this study, the OS and TTTF according to BCLC staging system were also analyzed. The subgroup analysis consisted of 219 and 213 patients in the orantinib and placebo arms. Median OS was 32.5 vs 33.0 months $(p=0.906)$, median TTP was 4.7 vs 3.1 months $(p=0.011)$, and median TTTF was 25.3 vs 18.2 months $(p=0.160)$ in the orantinib and placebo groups, respectively. Patients with BCLC-B in the orantinib and placebo groups showed a median OS of 33.7 and 30.1 months, respectively $(p=0.260)$, while the corresponding median TTTF were 25.3 and 14.0 months $(p=0.125)$. The Japanese population safety profile was similar to all over population in the ORIENTAL study. No significant differences were observed in the OS and TTTF though the TTP was significantly improved in the orantinib arm. The OS and TTTF showed a tendency to be prolonged following orantinib treatment of Japanese HCC patients with BCLC-B in the ORIENTAL study.
\end{abstract}

Keywords Angiogenic factors · Molecular targeted agents · Platelet-derived growth factor · Unresectable HCC · Vascular endothelial growth factor

\section{Introduction}

Hepatocellular carcinoma (HCC) is the fifth most common malignant neoplasm in men and the ninth most common in women worldwide. It is ranked second among the causes of malignant neoplasm-related deaths in both men and women [1]. Several therapies such as hepatic resection,

Electronic supplementary material The online version of this article (https://doi.org/10.1007/s12032-019-1272-2) contains supplementary material, which is available to authorized users.

Hisashi Hidaka

hisashi7@kitasato-u.ac.jp

Extended author information available on the last page of the article radiofrequency ablation, and percutaneous ethanol injection are indicated in the early stage of HCC. Transcatheter arterial chemoembolization (TACE) has been recognized as a treatment option for patients with intermediate HCC [2-4] and has been shown to improve patient survival rates $[5,6]$. In this procedure, antitumor drugs and an oil-based contrast medium are infused into a tumor vessel, which is then embolized with an embolic material. It has been demonstrated to improve the prognosis of unresectable $\mathrm{HCC}$ by inducing necrosis of intrahepatic tumors $[5,6]$. However, the recurrence rate of intrahepatic lesions after TACE has been reported as $37 \%$ and $61 \%$ at 6 and 12 months, respectively [7]. Therefore, recurrence after TACE is one of the most critical problems in these patients. 
Various angiogenic factors are involved in tumor recurrence after TACE [8]. In particular, the expression of vascular endothelial growth factor (VEGF) is closely associated with acute deterioration and metastases of tumors [9-13]. Furthermore, VEGF has been reported to increase significantly after TACE [14], and to lead to recurrent HCC and poor prognosis [15]. These reports suggest that the oral administration of molecular targeted drugs with VEGFinhibiting effects immediately after TACE may inhibit the deterioration of HCC after TACE. In recent years, combination therapy with molecular targeted drugs inhibiting the VEGF receptor to improve the therapeutic effects of TACE and adjuvant chemotherapy has been investigated as strategies to inhibit the recurrence of intrahepatic lesion and extra-hepatic metastasis and inconsistent results were reported. Although there were no differences on time to tumor progression in SPACE trial [16] combining of TACE with Sorafenib, progression free survival (PFS) in TACE2 [17] with Sorafenib, and overall survival [OS] in Brivanib (BRISK-TA [18]), Sorafenib in combination with TACE significantly improved PFS in TACTICS trial ( $\mathrm{HR}=0.59[95 \%$ CI 0.41-0.87], $p=0.006$ ) [19].

Orantinib (TSU-68) is a molecular targeted drug that inhibits VEGF, platelet-derived growth factor, and fibroblast growth factor receptors, and suppresses angiogenesis in tumor tissues. A phase III study of orantinib combined with TACE in patients with HCC (ORIENTAL) was conducted to verify if coadministration of TACE and orantinib inhibits the deterioration of HCC, and the OS in the orantinib arm was prolonged [20]. Patients were enrolled in Japan, Korea, and Taiwan. The interim analysis was aimed at evaluation of futility using the independent data monitoring committee (IDMC) stipulated in advance in the protocol. And the result of interim analysis, the trial was discontinued based on the recommendation of the IDMC. Prolongation of the OS was not demonstrated by the data analysis up to discontinuation of the trial. However, the TACE period in the orantinib arm was significantly longer than that in the placebo arm in patients with low vascular endothelial growth factor-C (VEGF-C) values. Subgroup analysis of TTTF revealed favor outcome for HCC patients with Barcelona Clinic of Liver Cancer (BCLC) stage B (BCLC-B).

The results of the ORIENTAL study suggest that the analysis of a population with less background variation would possibly provide a more detailed efficacy and safety evaluation of orantinib. Therefore, in this study, a subgroup analysis was conducted in the Japanese patients enrolled in the ORIENTAL study, who constituted the largest population.

\section{ORIENTAL Study}

The ORIENTAL study was a randomized, double-blind, placebo-controlled study of orantinib in patients with unresectable HCC [20]. After informed consent had been obtained, the patients were randomly assigned to the orantinib or placebo arms at a 1:1 ratio. Then, TACE was repeated until the criteria for discontinuation were met, and the study drug was administered during that period (Supplementary Fig. 1). The study was conducted in three regions, Japan, Korea, and Taiwan, and 889 patients were enrolled (445 and 444 patients in the orantinib and placebo arms, respectively) between December 2010 and November 2013. The primary endpoint was OS, and time to progression (TTP) and time to TACE failure (TTTF) were set as a secondary endpoint. Each endpoint, OS, TTP and TTTF were defined as the period from randomization to death, the period from the date of enrollment to that of the first observation of progressive disease, and the period from enrollment to the determination of TACE discontinuation, respectively. In this study, the patients were required to satisfy one of the following eligibility criteria regarding the number of tumors and tumor size; (a) The number of viable lesions was $\geq 4$, and at least one had a diameter $<1 \mathrm{~cm}$; (b) The number of viable lesions was 2 or 3 , and at least one had a diameter of $>3 \mathrm{~cm}$; (c) The patient had 1 viable lesion, which had a diameter $>5 \mathrm{~cm}$. The other major eligibility criteria were appropriate renal and hepatic functions (Child-Pugh class A), and the patients had a performance status (PS) of 0 or 1 . The stratification factors consisted of region (Japan, Korea, and Taiwan), alpha-fetoprotein (AFP) level ( $\leq 400 \mathrm{ng} / \mathrm{mL}$ vs $>400 \mathrm{ng} / \mathrm{mL})$, size of the largest nodule ( $\leq 5 \mathrm{~cm}$ vs $>5 \mathrm{~cm}$ ), and Child-Pugh score (5 vs 6 ). The enrollment of 889 patients was completed based on the original plan. No significant differences were observed in the OS among the three regions (31.1 for the orantinib arm vs 32.3 months for the placebo arm, $p=0.435$ ). On the other hand, the TTP was significantly prolonged (2.9 for the orantinib arm vs 2.5 months for the placebo arm, $p=0.0356$ ). In addition, the TTTF was tend to be longer, but with no statistical significance (23.9 for the orantinib arm vs 19.8 months for the placebo arm, $p=0.245$ ). The result of the regional analyses revealed that the hazard ratio (HR) of the OS of orantinib vs that of placebo was 0.981 (95\% confidence interval [CI] 0.717-1.343, $p=0.906$ ) in Japan, 1.318 (95\% CI 0.921-1.887, $p=0.129$ ) in Korea, and 0.984 (95\% CI 0.576-1.680, $p=0.953)$ in Taiwan. The HR of the TTTF of orantinib vs that of the placebo was 0.818 (95\% CI 0.618-1.083, $p=0.160)$ in Japan, 0.961 (95\% CI $0.681-1.355, p=0.819)$ in Korea, and 0.996 (95\% CI $0.573-1.730, p=0.988$ ) in Taiwan. The results of the subgroup analysis showed significant prolongation of TTTF in the orantinib arm in patients with low levels of VEGF$\mathrm{C}(\mathrm{HR}=0.695$ [95\% CI $0.512015-0.943$ ], $p=0.0196)$. Patients with BCLC-B might have better TTTF after treated by orantinib (HR $=0.764$ [95\% CI $0.580-1.005$ ], $p=0.055)$. This study was conducted in compliance with 
the Declaration of Helsinki (October 1996), the revised version, as well as the International Council for Harmonisation Guideline for Good Clinical Practice (Trial registration number in ClinicalTrials.gov: NCT01465464).

\section{Materials and methods}

\section{Patients}

The details of patient eligibility criteria have been reported previously [20]. In brief, patients with unresectable HCC, no extra-hepatic tumor spread, and Child-Pugh score of 6 or less were eligible.

\section{Study treatment and TACE}

As described previously [20], the study drug (orantinib, $200 \mathrm{mg}$ ) or the placebo was orally administered twice daily after meals. The dose could be reduced by up to two stages in response to adverse events and could be increased again up to the initial dose. The patients continued the study treatment until the criteria for discontinuation of TACE or study drug administration (such as adverse events) were met.

Concurrent use of embolization materials other than ethiodized oil such as gelatin sponges, porous gelatin particles, and drug-eluting beads, and use of antitumor drugs were essential when TACE was performed. In this trial, subsequent TACE was performed when the TACE re-performing criteria were met. The subsequent TACE was discontinued when the TACE discontinuation criteria were met. The TACE re-performing criteria were as follows: (a) evidence of incomplete tumor necrosis; (b) local tumor recurrence; and (c) evidence of new intrahepatic lesions. The discontinuation criteria for TACE were as follows: (a) the intrahepatic lesion could not be controlled by repeated TACE; (b) the target vessel was damaged and a catheter could not be used for the feeding vessel; (c) additional TACE was difficult because of the emergence of vascular invasion; (d) extra-hepatic metastasis had occurred, and treatment other than TACE was deemed necessary; and (e) the hepatic function had been exacerbated to the Child-Pugh classification C persistently for 28 days.

\section{Assessments}

Eligible patients who had only one tumor with a size $\geq 5 \mathrm{~cm}$ were classified as BCLC stage A (BCLC-A); those with a PS 0 without portal invasion who had multiple lesions (two or three lesions of $\geq 3 \mathrm{~cm}$ or four lesions or more) were classified as BCLC-B; and patients with portal invasion and a PS of 1 were classified as BCLC stage C (BCLC-C). The vital signs and body weight were examined, and the Eastern
Cooperative Oncology Group (ECOG) PS, Child-Pugh score, and clinical chemistry and hematology tests were conducted at the time of screening and 14,28, and 42 days after the initial (subsequent) TACE, and every 28 days after that. A safety follow-up test was conducted 30 days after the last study drug administration. Image analysis of the tumors was conducted within 28 days before the initial TACE. After the study drug administration had commenced, it was conducted 42 days after the initial (subsequent) TACE, and every 56 days after that. Concerning safety, all adverse events (AEs) and adverse drug reactions that occurred between the day the study drug administration commenced and the completion day of the observation in the clinical trial period were counted. All the AEs were recorded in the electronic case report form (eCRF) using the terminology of the Common Terminology Criteria for Adverse Events Ver. 4.02.

\section{Statistical analysis}

Subgroup analysis was conducted for Japanese patients in the full analysis set (FAS) of the ORIENTAL study. The OS, TTP, and TTTF of the orantinib arm were compared with those of the placebo arm using an unstratified log-rank test. The survival curves of the OS, TTP, and TTTF were constructed using the Kaplan-Meier method, and the median and 95\% CI were calculated. The OS and TTTF according to BCLC staging system were also analyzed.

Concerning safety, the AE and adverse drug reaction incidences were calculated for the FAS. The statistical analysis software (SAS, version 9.2, SAS Institute Inc, Cary, NC), was used for all analyses. All reported $p$-values are twosided and $p<0.05$ was considered statistically significant.

\section{Results \\ Patients}

A total of 432 Japanese patients (FAS; 219 and 213 in the orantinib and placebo arms, respectively) were included in this analysis (Fig. 1 and Table 1). The breakdown of the BCLC stage was as follows: BCLC-A in 119 patients (65 and 54 in the orantinib and placebo arms, respectively), BCLC-B in 240 patients (121 and 119 in the orantinib and placebo arms, respectively, Supplementary Table 1), and BCLC-C in 57 patients (27 and 30 in the orantinib and placebo arms, respectively). Furthermore, 16 patients were in the alternate classification [15 in BCLC-0 (six and nine in the orantinib and placebo arms, respectively) and one in the unknown (in the placebo arm)]. Twenty-eight patients (15 and 13 in the orantinib and placebo arms, respectively) had portal invasion. 
Fig. 1 Allocation of patients and description of orantinib and placebo groups

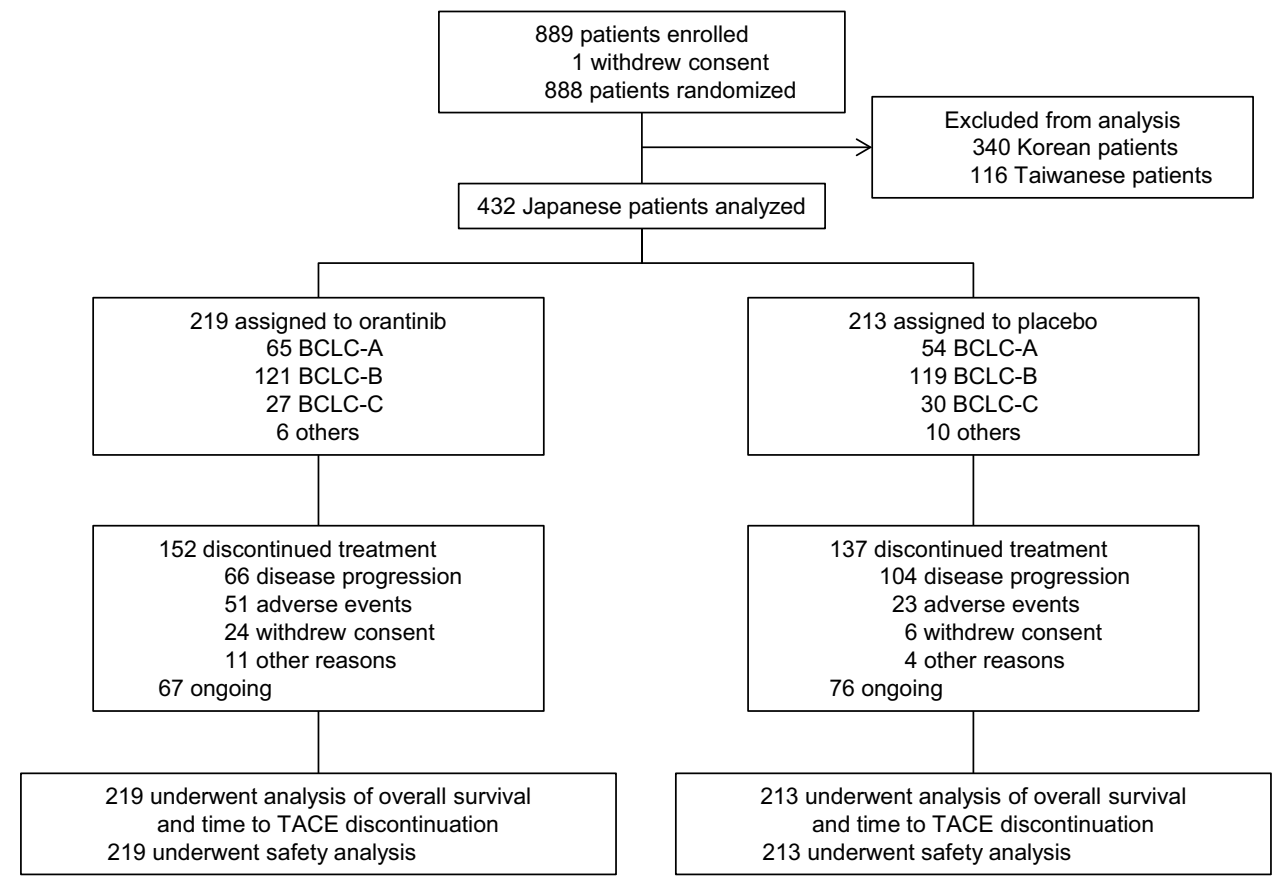

\section{Study treatment}

The median duration of treatment with the study drug was 298 and 354 days in the orantinib and placebo arms, respectively, indicating that the treatment duration in the orantinib arm was shorter. The proportion of patients who required dose reductions was $49.8 \%$ and $11.7 \%$ in the orantinib and placebo arm, indicating that the proportion was higher in the orantinib arm.

\section{Efficacy}

Among the 432 patients included in the analysis, 77 and 79 in the orantinib and placebo arms died before the study was discontinued. The most common cause of death was the deterioration of HCC. The median OS was 32.5 and 33.0 months in the orantinib and placebo arms, respectively $(\mathrm{HR}=0.981$ [95\% CI 0.717-1.343], $p=0.906$, Fig. 2a). The median TTP was 4.7 months and 3.1 months in the orantinib and placebo arms, respectively ( $\mathrm{HR}=0.76[95 \%$ CI 0.619-0.940], $p=0.011$, Fig. 2b). The median TTTF was 25.3 and 18.2 months in the orantinib and placebo arms, respectively (HR, $0.818, p=0.160$, Fig. $2 c$ ). The OS and TTTF, as classified by the BCLC stage, is shown in Fig. 3. BCLC-C included patients who had portal invasion or a PS of 1 . When these groups were combined, and the patients with and without portal invasion were compared, the median TTTF was 9.4 and 27.5 months $(n=28$ and 29) in patients with and without portal invasion, respectively $(\mathrm{HR}=2.820$ [95\% CI 1.347-5.902], $p=0.0041)$. In addition, the median OS was 29.5 and 31.8 months in patients with and without portal invasion, respectively (HR $=1.519[95 \%$ CI 0.706-3.264], $p=0.2814$ ).

\section{Interval of each TACE session}

The intervals between the first and second TACE sessions and that between the second and third sessions were regarded as Intervals 1 and 2, respectively, and the designation continued in this manner up to Interval 4. Orantinib treatment tended to prolong the interval between each TACE session. When the orantinib and the placebo arms were compared, Interval 1 was 142.5 and 129.0 , Interval 2 was 136.0 and 131.0 days, Interval 3 was 126.0 and 117.0 days, and Interval 4 was 117.0 and 98.0 days, respectively (Table 2). According to the BCLC stage, all intervals for patients with BCLC$\mathrm{B}$ tended to be prolonged by approximately 7 to 34 days (median) in the orantinib arm, but no consistent tendency was observed for patients with BCLC-A and BCLC-C.

\section{Safety}

The AEs observed in the orantinib and placebo arms are listed in Table 3. Most adverse events were of grade 1 or 2 . The most frequently reported adverse events in the orantinib group were pyrexia $(74.4 \%$ in the orantinib arm vs $84.0 \%$ in the placebo arm), The most frequent $\geq$ grade 3 adverse events were AST increased $63 \%$ in the orantinib arm vs $57.3 \%$ in the placebo arm) and ALT increased $(47.5 \%$ vs $43.3 \%$ ). The incidence of hypertension was $16.4 \%$ in the orantinib arm and $16.9 \%$ in the placebo arm. The AEs with an incidence $>20 \%$ in the orantinib arm and $10 \%$ higher than 
Table 1 Patient background

\begin{tabular}{|c|c|c|}
\hline & $\begin{array}{l}\text { Orantinib }(N=219) \\
n(\%)\end{array}$ & $\begin{array}{l}\text { Placebo }(N=213) \\
n(\%)\end{array}$ \\
\hline \multicolumn{3}{|l|}{ Sex } \\
\hline Male & $178(81.3)$ & $176(82.6)$ \\
\hline Female & $41(18.7)$ & $37(17.4)$ \\
\hline \multicolumn{3}{|l|}{ Age (years) } \\
\hline Median (range) & $71.0(44-86)$ & $71.0(46-87)$ \\
\hline \multicolumn{3}{|l|}{ ECOG PS } \\
\hline 0 & $207(94.5)$ & $195(91.5)$ \\
\hline 1 & $12(5.5)$ & $18(8.5)$ \\
\hline \multicolumn{3}{|l|}{ Stage of HCC } \\
\hline I & $1(0.5)$ & $0(0.0)$ \\
\hline II & $92(42.0)$ & $84(39.4)$ \\
\hline III & $115(52.5)$ & $118(55.4)$ \\
\hline IVA & $11(5.0)$ & $11(5.2)$ \\
\hline IVB & $0(0.0)$ & $0(0.0)$ \\
\hline \multicolumn{3}{|l|}{ BCLC staging $^{\mathrm{a}}$} \\
\hline 0 & $6(2.7)$ & $9(4.2)$ \\
\hline $\mathrm{A}$ & $65(29.7)$ & $54(25.4)$ \\
\hline $\mathrm{B}$ & $121(55.3)$ & $119(55.9)$ \\
\hline $\mathrm{C}$ & $27(12.3)$ & $30(14.1)$ \\
\hline \multicolumn{3}{|l|}{ Child-Pugh score } \\
\hline 5 & $148(67.6)$ & $145(68.1)$ \\
\hline 6 & $71(32.4)$ & $68(31.9)$ \\
\hline \multicolumn{3}{|l|}{ Prior TACE } \\
\hline Yes & $109(49.8)$ & $116(54.5)$ \\
\hline \multicolumn{3}{|l|}{ HBsAg } \\
\hline Positive & $39(17.8)$ & $30(14.1)$ \\
\hline Negative & $180(82.2)$ & $183(85.9)$ \\
\hline \multicolumn{3}{|l|}{$\mathrm{HCVAb}^{\mathrm{a}}$} \\
\hline Positive & $131(59.8)$ & $122(57.3)$ \\
\hline Negative & $88(40.2)$ & $91(42.7)$ \\
\hline \multicolumn{3}{|l|}{$\operatorname{AFP}(n g / m L)^{a}$} \\
\hline Median (range) & $22.0(1.1-16956.0)$ & $22.5(0.0-32200.0)$ \\
\hline
\end{tabular}

Reprinted from The Lancet Gastroenterology and Hepatology, Vol. 3, Kudo $\mathrm{M}$ et al. Orantinib versus placebo combined with transcatheter arterial chemoembolization in patients with unresectable hepatocellular carcinoma (ORIENTAL): a randomized, double-blind, placebocontrolled, multicentre, phase 3 study, 37-46, Appendix Copyright (2018), with permission from Elsevier

ECOG PS Eastern Cooperative Oncology Group performance status, $H C C$ hepatocellular carcinoma, $B C L C$ Barcelona Clinic of Liver Cancer, TACE transcatheter arterial chemoembolization, HBsAg hepatitis B surface antigen, $H C V A b$ hepatitis $\mathrm{C}$ antibody

${ }^{a}$ Data were unknown for some patients

those in the placebo arm were impaired appetite, chromaturia, edema of the extremities, ascites, diarrhea, and facial edema. The events with severity $\geq$ grade 3 and that occurred with an incidence $\geq 10 \%$ in the orantinib arm were increased AST, ALT, and lymphocytes; hypertension; and decreased platelet. The proportion of patients who experienced serious
AEs was $45.2 \%$ and $27.2 \%$ in the orantinib and placebo arms, respectively, indicating that the incidence was higher in the orantinib arm. No study drug-related deaths occurred. The proportion of treatment discontinuation related to AEs was $33.6 \%(51 / 152)$ in the orantinib arm vs $16.8 \%(23 / 137)$ in the placebo arm (hereinafter in this order) in all Japanese patients, $38.5 \%(15 / 39)$ vs $14.8 \%(4 / 27)$ in patients with BCLC-A, 32.6\% (30/92) vs 14.5\% (12/83) with BCLC-B, and $31.6 \%(6 / 19)$ vs $13.6 \%(3 / 22)$ with BCLC-C.

\section{Discussion}

In the subgroup analysis of the Japanese population of HCC patients in the ORIENTAL study, there observed significant longer TTP in the orantinib arm than in the placebo arm. Hence the result of PFS improvement was observed in the former phase II study [21], similar result of the TTP prolongation was reproduced in the ORIENTAL study. Despite the significant improvement of TTP, it is failed to observe significant differences of the OS and the TTTF in Japanese patients with HCC.

Since the TTP shows the duration until first progression, significant difference in TTP suggested that orantinib could prevent progression of intrahepatic lesions or extra-hepatic metastasis for certain period. On the other hand, the TTTF presenting the time to TACE failure and the OS were not improved by addition of orantinib. It is hard to specify the reasons for no clinically significance but the efficacy of orantinib might be not enough to prolong the TTTF and the OS. In addition, safety profile of the combination therapy of orantinib and TACE was possibly unfavorable because the percentage of discontinuation of study drug administration due to adverse events and the dose reduction rate were $33.6 \%$ and $16.8 \%$, and was $49.8 \%$ and $11.7 \%$ in the orantinib and placebo arms, respectively. As a result, the benefits from combination therapy were probably discounted.

Although no significant differences were observed in the OS and TTTF, subgroup analysis showed that difference of classification of BCLC was likely to have been related to the efficacy of combination therapy of orantinib and TACE. Especially in patient with BCLC-B, there were tended to be prolonged not only the TTTF but also the OS in the orantinib arm. The patients stratified as BCLC-A to C were enrolled based on the clinical situation in Asia where TACE is performed for patients stratified as BCLC-A to C [22]. However, treatment for $\mathrm{HCC}$ is provided according to BCLC in Europe and the US, and BCLC-B is recommended as the target for TACE $[2,23]$. When the patients were limited to the BCLC-B stage in this analysis, each TACE interval tended to be prolonged by taking with orantinib. Orantinib was thought to have contributed to the prolongation of the period until discontinuation of TACE and the survival period 
A

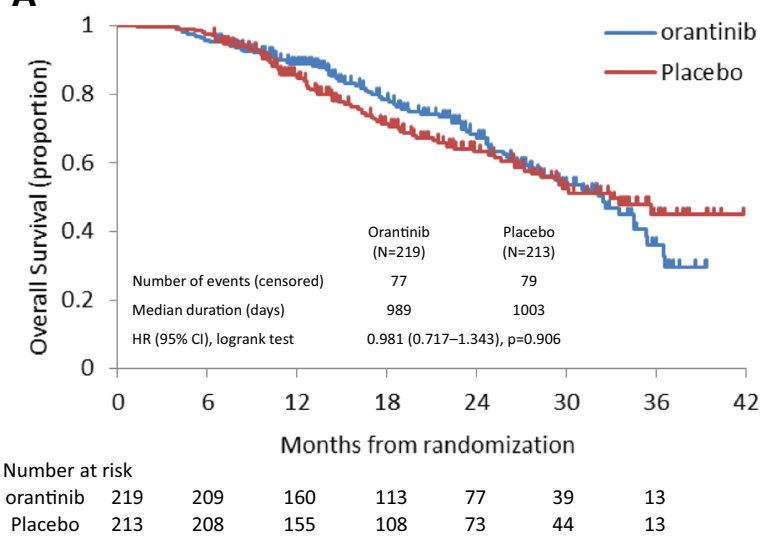

B

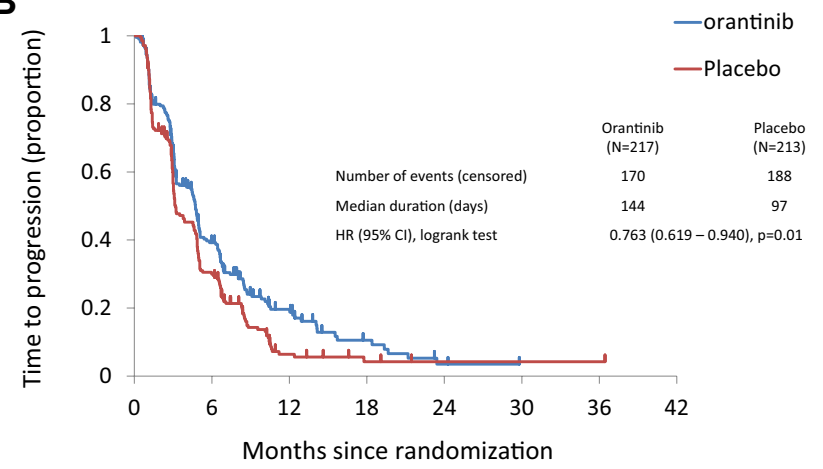

Number at risk

$\begin{array}{cccccccc}\text { Orantinib } & 217 & 73 & 24 & 8 & 2 & - & - \\ \text { Placebo } & 213 & 61 & 8 & 3 & 1 & 1 & 1\end{array}$

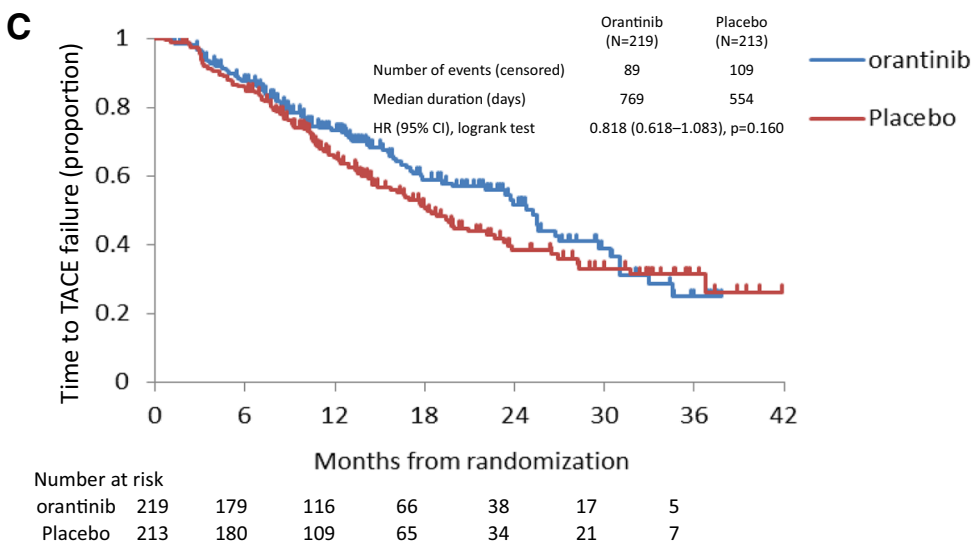

Fig. 2 Kaplan-Meier curve for overall survival (OS) (a), time to progression (TTP) (b) and time to transcatheter arterial chemoembolization (TACE) failure (TTTF) (c). a, c is reprinted from The Lancet Gastroenterology and Hepatology, Vol. 3, Kudo M et al. Orantinib versus placebo combined with transcatheter arterial chemoemboliza- tion in patients with unresectable hepatocellular carcinoma (ORIENTAL): a randomized, double-blind, placebo-controlled, multicentre, phase 3 study, 37-46, Appendix, Copyright (2018), with permission from Elsevier

with portal invasion, rather than sorafenib, which inhibits VEGF similar to orantinib [25]. Furthermore, another report indicates that TACE did not have any advantage in patients with portal invasion who were cotreated with sorafenib and TACE [26]. This observation suggests that patients with portal invasion were not appropriate subjects for evaluating additional molecular targeted drugs in combination with TACE. In patients with portal invasion, the generation of resistance in the portal blood flow decreases the blood flow to the liver via the portal vein and, thereby, exacerbates the hepatic dysfunction. Therefore, it is believed that patients with portal invasion were inappropriately treated with TACE, which potentially damages the hepatic parenchyma [27]. Moreover, inhibition of VEGF reduces the portal blood flow volume and exacerbates hepatic functions [28]. In addition, in this study, the administration of orantinib is thought to have worsened the patients' hepatic functions. Further studies and analyses are considered necessary to determine 
$B C L C-A$ OS

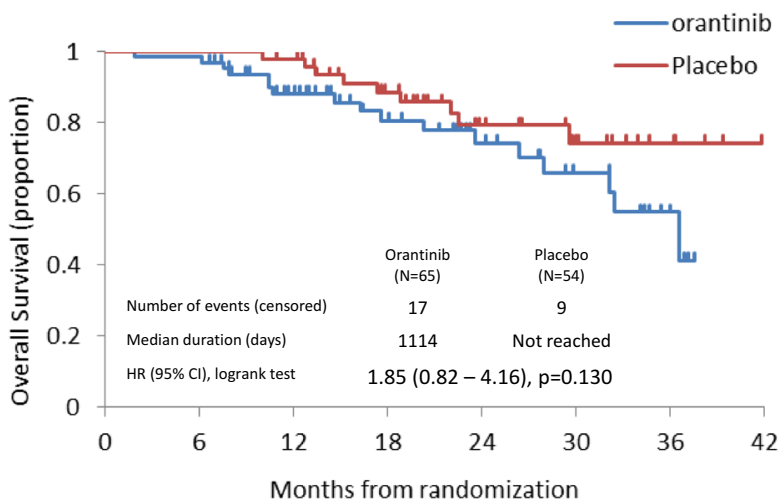

Number at risk $\begin{array}{cccccccc}\text { orantinib } & 65 & 63 & 44 & 31 & 21 & 13 & 5 \\ \text { Placebo } & 54 & 54 & 46 & 34 & 22 & 14 & 5\end{array}$

$B C L C-B$ OS

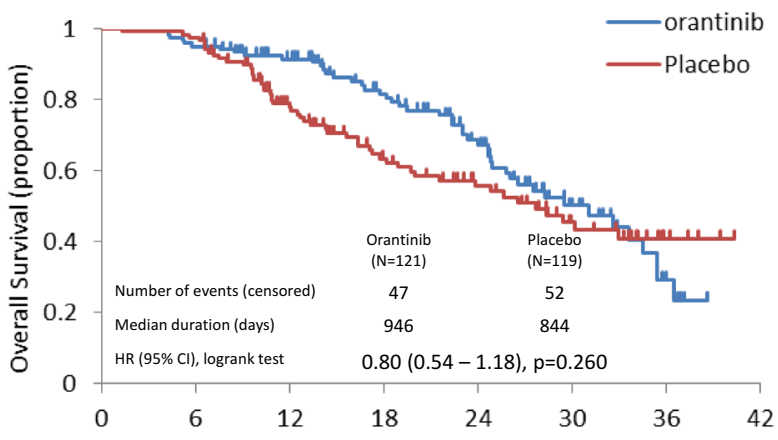

Months from randomization

Number at risk orantinib 121 Placebo 119
BCLC-A TTTF

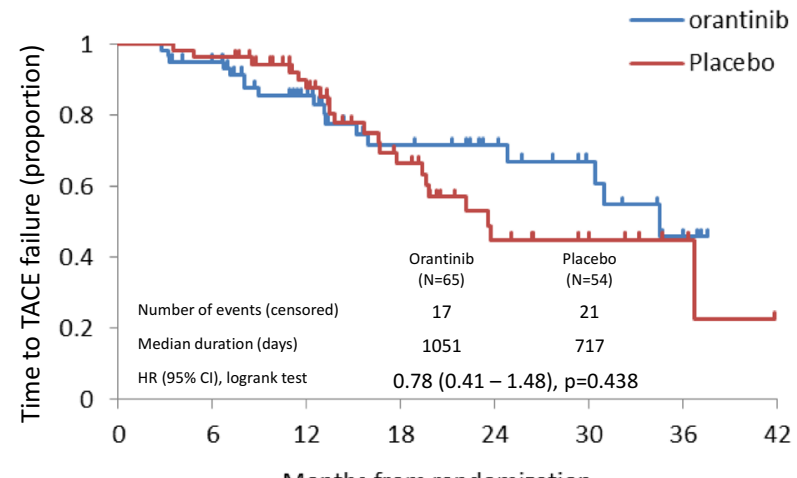

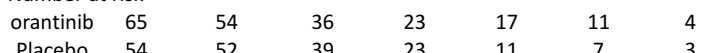

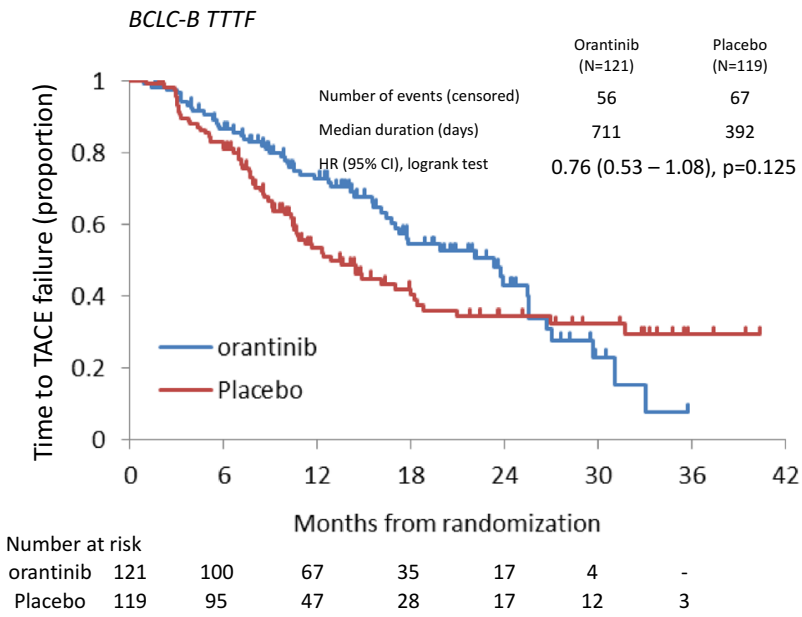

BCLC-C TTTF

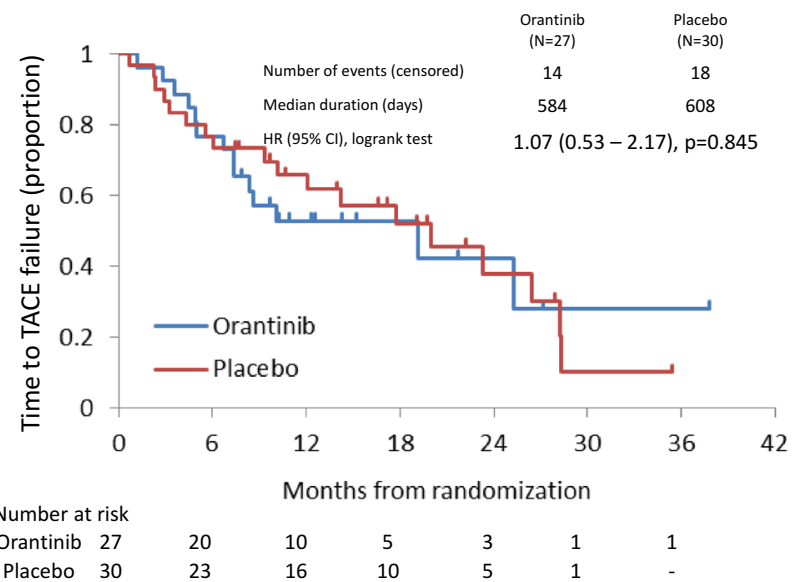

Fig. 3 Kaplan-Meier curve for overall survival (OS) and time to transcatheter arterial chemoembolization (TACE) failure (TTTF) based on Barcelona Clinic of Liver Cancer (BCLC) staging 
Table 2 TACE interval

\begin{tabular}{|c|c|c|c|c|c|c|c|c|c|c|c|c|c|}
\hline \multirow[b]{3}{*}{ BCLC } & \multirow[b]{3}{*}{ Arm } & \multirow{2}{*}{\multicolumn{3}{|c|}{$\frac{\text { Interval } 1}{1 \text { st-2nd }}$}} & \multirow{2}{*}{\multicolumn{3}{|c|}{$\frac{\text { Interval } 2}{2 \text { nd-3rd }}$}} & \multirow{2}{*}{\multicolumn{3}{|c|}{$\frac{\text { Interval } 3}{3 \text { rd-4th }}$}} & \multirow{2}{*}{\multicolumn{3}{|c|}{$\frac{\text { Interval } 4}{4 \text { th-5th }}$}} \\
\hline & & & & & & & & & & & & & \\
\hline & & $n$ & $\%$ & Median (days) & $n$ & $\%$ & Median (days) & $n$ & $\%$ & Median (days) & $n$ & $\%$ & Median (days) \\
\hline \multicolumn{14}{|l|}{ All } \\
\hline & Orantinib & 154 & 70.3 & 142.5 & 87 & 56.5 & 136.0 & 50 & 57.5 & 126.0 & 26 & 52.0 & 117.0 \\
\hline & Placebo & 177 & 83.1 & 129.0 & 118 & 66.7 & 131.0 & 73 & 61.9 & 117.0 & 44 & 60.3 & 98.0 \\
\hline \multicolumn{14}{|l|}{$\mathrm{O}$} \\
\hline & Orantinib & 6 & 100.0 & 184.5 & 2 & 33.3 & 235.5 & 0 & & & 0 & & \\
\hline & Placebo & 7 & 77.8 & 203.0 & 4 & 57.1 & 159.0 & 2 & 50.0 & 87.5 & 2 & 100.0 & 98.0 \\
\hline \multicolumn{14}{|l|}{ A } \\
\hline & Orantinib & 37 & 56.9 & 169.0 & 23 & 62.2 & 132.0 & 15 & 65.2 & 160.0 & 9 & 60.0 & 105.0 \\
\hline & Placebo & 47 & 87.0 & 162.0 & 33 & 70.2 & 175.0 & 24 & 72.7 & 126.5 & 14 & 58.3 & 110.5 \\
\hline \multicolumn{14}{|l|}{ B } \\
\hline & Orantinib & 88 & 72.7 & 140.5 & 55 & 62.5 & 139.0 & 32 & 58.2 & 107.0 & 15 & 46.9 & 119.0 \\
\hline & Placebo & 98 & 82.4 & 119.0 & 65 & 66.3 & 119.0 & 36 & 55.4 & 100.5 & 20 & 55.6 & 85.5 \\
\hline \multicolumn{14}{|l|}{$\mathrm{C}$} \\
\hline & Orantinib & 23 & 85.2 & 133.0 & 7 & 30.4 & 105.0 & 3 & 42.9 & 70.0 & 2 & 66.7 & 153.0 \\
\hline & Placebo & 24 & 80.0 & 124.0 & 15 & 62.5 & 129.0 & 10 & 66.7 & 113.0 & 7 & 70.0 & 91.0 \\
\hline
\end{tabular}

Reprinted from The Lancet Gastroenterology and Hepatology, Vol. 3, Kudo M et al. Orantinib versus placebo combined with transcatheter arterial chemoembolization in patients with unresectable hepatocellular carcinoma (ORIENTAL): a randomized, double-blind, placebo-controlled, multicentre, phase 3 study, 37-46, Appendix, Copyright (2018), with permission from Elsevier

Table 3 All-grade adverse events with frequency $>20 \%$ and grade 3 or higher adverse events with frequency $>10 \%$ in either group in the safety population

\begin{tabular}{|c|c|c|c|c|}
\hline \multirow[t]{2}{*}{ Adverse events } & \multicolumn{2}{|c|}{ Orantinib $(N=219)$} & \multicolumn{2}{|c|}{ Placebo $(N=213)$} \\
\hline & All grade $N(\%)$ & $\begin{array}{l}\text { Grade } \geq 3 \\
N(\%)\end{array}$ & All grade $N(\%)$ & $\begin{array}{l}\text { Grade } \geq 3 \\
N(\%)\end{array}$ \\
\hline Pyrexia & $163(74.4)$ & $1(0.5)$ & $179(84.0)$ & $3(1.4)$ \\
\hline AST increased & $151(68.9)$ & $138(63.0)$ & $134(62.9)$ & $122(57.3)$ \\
\hline Abdominal pain & $142(64.8)$ & $5(2.3)$ & $130(61.0)$ & $1(0.5)$ \\
\hline ALT increased & $132(60.3)$ & $104(47.5)$ & $110(51.6)$ & $90(42.3)$ \\
\hline Decreased appetite & $110(50.2)$ & $10(4.6)$ & $80(37.6)$ & $7(3.3)$ \\
\hline Constipation & $96(43.8)$ & $1(0.5)$ & $86(40.4)$ & 0 \\
\hline Chromaturia & $90(41.1)$ & 0 & $18(8.5)$ & 0 \\
\hline Oedema peripheral & $87(39.7)$ & $1(0.5)$ & $40(18.8)$ & 0 \\
\hline Nausea & $85(38.8)$ & 0 & $98(46.0)$ & $1(0.5)$ \\
\hline Malaise & $81(37.0)$ & $2(0.9)$ & $76(35.7)$ & 0 \\
\hline Ascites & $80(36.5)$ & $7(3.2)$ & $44(20.7)$ & $5(2.3)$ \\
\hline Diarrhea & $78(35.6)$ & $4(1.8)$ & $35(16.4)$ & $5(2.3)$ \\
\hline Vomiting & $73(33.3)$ & $2(0.9)$ & $65(30.5)$ & 0 \\
\hline Face oedema & $67(30.6)$ & $2(0.9)$ & $5(2.3)$ & 0 \\
\hline Hypoalbuminemia & $64(29.2)$ & $5(2.3)$ & $56(26.3)$ & $2(0.9)$ \\
\hline Back pain & $61(27.9)$ & $2(0.9)$ & $67(31.5)$ & $1(0.5)$ \\
\hline Upper respiratory tract infection & $47(21.5)$ & 0 & $53(24.9)$ & $1(0.5)$ \\
\hline C-reactive protein increased & $46(21.0)$ & $2(0.9)$ & $43(20.2)$ & $2(0.9)$ \\
\hline Hypertension & $36(16.4)$ & $29(13.2)$ & $36(16.9)$ & $25(11.7)$ \\
\hline Lymphocyte count decreased & $35(16.0)$ & $31(14.2)$ & $41(19.2)$ & $39(18.3)$ \\
\hline Platelet count decreased & $35(16.0)$ & $28(12.8)$ & $32(15.0)$ & $19(8.9)$ \\
\hline
\end{tabular}


whether BCLC-C patients are appropriate subjects for the study of the coadministration of TACE and molecular targeted drugs.

Concerning safety, AEs of all grades with incidences that were higher than $20 \%$ or more in the orantinib arm than in the placebo arm were abdominal pain, ascites, constipation, nausea, vomiting, malaise, pyrexia, upper respiratory tract infection, increased ALT, increased AST, increased C-reactive protein, impaired appetite, decreased albumin, and back pain. The AEs associated with fluid retention, such as edema of the extremities, facial edema, and ascites were common in the orantinib arm, similar to the safety profile of the three regions. The grade 3 or higher AEs included increased AST and ALT. Other AEs of grade 3 or higher and those that were $5 \%$ higher in the orantinib arm than in the placebo arm were not observed. Moreover, deaths related to the study drug did not occur, which demonstrated the tolerability of orantinib in Japanese people.

By limiting the subgroup analysis to the Japanese population, we minimized the heterogeneity of various patient backgrounds. A tendency for TTTF prolongation in patients was observed in the orantinib arm regardless of the BCLC staging. In particular, a tendency for prolongation of OS and TTTF in patients was observed in the orantinib arm for the BCLC-B stage. Forest plot of PFS divided by BCLC stage A or B in TACTICS also showed similar result that significant improvement were only observed in BCLC-B patients. Patients in the BCLC-A and BCLC-C stage were suggested to be inappropriate for the TACE combination study with an antiangiogenic agent because TACE alone was effective for BCLC-A. In addition, the evaluation of the study drug efficacy in combination with TACE was difficult because the BCLC-C patients with portal invasion showed a significantly worse TTTF and OS, which have considerable effects on the evaluation of the study drug efficacy. We believe that it is important for studies investigating antiangiogenic agents in combination with TACE to limit the study patients to the BCLC-B stage. Furthermore, it is important to appropriately manage the AEs to properly evaluate the effects of a study drug.

In conclusion, although the TTP was significantly improved in the orantinib arm, no significant differences were observed in the OS and TTTF. The orantinib arm of the BCLC-B stage of Japanese patients in the ORIENTAL study tended to show a prolongation of the OS and TTTF. This subgroup analysis suggested that further studies should be conducted to identify appropriate patients for recruitment as study subjects in future TACE combination trials, including studies of other molecular targeted drugs.

Acknowledgements We thank all the patients, their families, and the investigators who participated in the study. We thank Yuji Matsuda, Taiho Pharmaceutical for his support in compiling and writing this report. The study was designed by Taiho Pharmaceutical in conjunction with the principal academic investigator. Data collection was performed by EPS. BSR Corporation performed the statistical analysis of the collected data. Data were managed in parallel by the study's sponsor and its principal investigator. This study was sponsored by Taiho Pharmaceutical Co., Ltd.

Data availability Data generated or analyzed during this study are on file with Taiho Pharmaceutical Co., Ltd. and not publicly available. Inquiries for data access may be sent to the following e-mail address: TOIAWASE@taiho.co.jp.

\section{Compliance with ethical standards}

Conflicts of interest statement Hidaka $\mathrm{H}$ has received personal fees from Taiho Pharmaceutical. Izumi $\mathrm{N}$ has received personal fees from Gilead, AbbVie, Otsuka and Shionogi. Aramaki T has received personal fees from Taiho. Ikeda $\mathrm{M}$ has received: personal fees from Taiho, Bayer, Eisai,Bristol-Myers Squibb, Eli Lilly Japan, Daiichi-Sankyo, Kyowa Hakko Kirin, Novartis Pharma, Yakult, Abbott Japan, Chugai Pharmaceutical, Nippon Kayaku, Otsuka, Pharmaceutical and BristolMyers Squibb. Ikeda M has received other from Taiho Pharmaceutical, Bayer Yakuhin, Eisai, Bristol-Myers Squibb, Eli Lilly Japan, DaiichiSankyo, Kyowa Hakko Kirin, TEIJIN PHARMA, Novartis Pharma, Shire, Chugai Pharmaceutical, Ono Pharmaceutical, AstraZeneca, Zeria Pharmaceutical, Baxter, Kowa, Boehringer Ingelheim, Glaxo Smithkline, Nano Carrier, ASLAN Pharmaceuticals. Inaba Y has received personal fees from Eisai, Medicon, Bayer Yakuhin, Guerbet Japan, Nippon Kayaku, Canon Medical Systems, Toray Medical. Okusaka T has received other from Merck Serono, Suimtomo Dainippon, Yakult, Ono, Novartis, Eli Lilly Japan, Chugai, AstraZeneca, Eisai and Baxter. Kaneko S has received other from Taiho. Furuse J has received: grant from Taiho, Yakult, Eli Lilly Japan, Chugai, Eisai, Ono, Kyowa Hakko Kirin, Sumitomo Dainippon, Astellas, MSD, J-Pharma, Daiichi Sankyo, Janssen, Zeria, Merck Serono, Onco Therapy Science, Bayer, Takeda, Shire, Pfizer, Bristol-Myers Squibb, Novartis, Astra Zeneca, Mochida and Shionogi; personal fees from Taiho, Yakult, Eli Lilly japan, Chugai, Eisai, Ono, Kyowa Hakko Kirin, Sumitomo Dainippon, Astellas, J-Pharma, Daiichi Sankyo, Zeria, Merck Serono, Bayer, Takeda, Shire, Pfizer, Bristol-Myers Squibb, Novartis, Astra Zeneca, Mochida, Shionogi, EA Pharma, Mitsubishi Tanabe, Sawai, Sanofy, Sandoz, Boehringer Ingelheim, Otsuka and Fujifilm. Yamashita T has received personal fees from Taiho Pharmaceutical. Yokosuka O has received other from Tanabe-Mitsubishi, Otsuka, Eisai, Daiichi Sankyo, MSD, Gilead, Chugai, Astellas, Takeda and Sumitomo Dainippon. Morita $\mathrm{S}$ has received personal fees from Taiho. Arioka $\mathrm{H}$ has received personal fees from Taiho and Chugai. Kudo $M$ has received: grant from Taiho, Chugai, Otsuka, Takeda, Sumitomo Dainippon, Daiichi Sankyo, MSD, Eisai, Bayer, Abbvie, Medico's Hirata, Astellas Pharma and Bristol-Myers Squibb; personal fees from Taiho, MSD, Eisai, Bayer and Ajinomoto. Arai Y has received personal fees from Taiho. All remaining authors have declared no conflicts of interest.

Ethical approval All procedures performed in studies involving human participants were in accordance with the ethical standards of the institutional and/or national research committee and with the 1964 Helsinki declaration and its later amendments or comparable ethical standards.

Informed consent Informed consent was obtained from all individual participants included in the study.

Open Access This article is distributed under the terms of the Creative Commons Attribution 4.0 International License (http://creat ivecommons.org/licenses/by/4.0/), which permits unrestricted use, 
distribution, and reproduction in any medium, provided you give appropriate credit to the original author(s) and the source, provide a link to the Creative Commons license, and indicate if changes were made.

\section{References}

1. Ferlay J, Soerjomataram I, Dikshit R, Eser S, Mathers C, Rebelo M, Parkin DM, Forman D, Bray F. Cancer incidence and mortality worldwide: sources, methods and major patterns in GLOBOCAN 2012. Int J Cancer. 2015;136:E359-86.

2. Bruix J, Sherman M. American Association for the Study of Liver Diseases. Management of hepatocellular carcinoma: an update. Hepatology. 2011;53:1020-2.

3. European Association for Study of Liver. European Organisation for Research and Treatment of Cancer. EASL-EORTC clinical practice guidelines: management of hepatocellular carcinoma. Eur J Cancer. 2012;48:599-641.

4. Kudo M, Izumi N, Kokudo N, Matsui O, Sakamoto M, Nakashima O, Kojiro M, Makuuchi M. HCC Expert Panel of Japan Society of Hepatology: management of hepatocellular carcinoma in Japan: Consensus-Based Clinical Practice Guidelines proposed by the Japan Society of Hepatology (JSH) 2010 updated version. Dig Dis. 2011;29:339-64.

5. Llovet JM, Real MI, Montaña X, Planas R, Coll S, Aponte J, Ayuso C, Sala M, Muchart J, Solà R, Rodés J, Bruix J. Barcelona Liver Cancer Group: arterial embolisation or chemoembolisation versus symptomatic treatment in patients with unresectable hepatocellular carcinoma: a randomised controlled trial. Lancet. 2002;359:1734-9.

6. Lo CM, Ngan H, Tso WK, Liu CL, Lam CM, Poon RT, Fan ST, Wong J. Randomized controlled trial of transarterial lipiodol chemoembolization for unresectable hepatocellular carcinoma. Hepatology. 2002;35:1164-71.

7. Terzi E, Golfieri R, Piscaglia F, Galassi M, Dazzi A, Leoni S, Giampalma E, Renzulli M, Bolondi L. Response rate and clinical outcome of HCC after first and repeated cTACE performed "on demand". J Hepatol. 2012;57:1258-67.

8. El-Assal ON, Yamanoi A, Soda Y, Yamaguchi M, Igarashi M, Yamamoto A, Nabika T, Nagasue N. Clinical significance of microvessel density and vascular endothelial growth factor expression in hepatocellular carcinoma and surrounding liver: possible involvement of vascular endothelial growth factor in the angiogenesis of cirrhotic liver. Hepatology. 1998;27:1554-62.

9. Mise M, Arii S, Higashituji H, Furutani M, Niwano M, Harada T, Ishigami S, Toda Y, Nakayama H, Fukumoto M, Fujita J, Imamura M. Clinical significance of vascular endothelial growth factor and basic fibroblast growth factor gene expression in liver tumor. Hepatology. 1996;23:455-64.

10. Torimura T, Sata M, Ueno T, Kin M, Tsuji R, Suzaku K, Hashimoto O, Sugawara $\mathrm{H}$, Tanikawa K. Increased expression of vascular endothelial growth factor is associated with tumor progression in hepatocellular carcinoma. Hum Pathol. 1998;29:986-91.

11. Li XM, Tang ZY, Zhou G, Lui YK, Ye SL. Significance of vascular endothelial growth factor mRNA expression in invasion and metastasis of hepatocellular carcinoma. J Exp Clin Cancer Res. 1998;17:13-7.

12. Poon RT, Lau CP, Ho JW, Yu WC, Fan ST, Wong J. Tissue factor expression correlates with tumor angiogenesis and invasiveness in human hepatocellular carcinoma. Clin Cancer Res. 2003;9:5339-45.

13. Schmitt M, Horbach A, Kubitz R, Frilling A, Häussinger D. Disruption of hepatocellular tight junctions by vascular endothelial growth factor (VEGF): a novel mechanism for tumor invasion. J Hepatol. 2004;41:274-83.

14. Suzuki H, Mori M, Kawaguchi C, Adachi M, Miura S, Ishii $\mathrm{H}$. Serum vascular endothelial growth factor in the course of transcatheter arterial embolization of hepatocellular carcinoma. Int J Oncol. 1999;14:1087-90.

15. Chao Y, Li CP, Chau GY, Chen CP, King KL, Lui WY, Yen SH, Chang FY, Chan WK, Lee SD. Prognostic significance of vascular endothelial growth factor, basic fibroblast growth factor, and angiogenin in patients with resectable hepatocellular carcinoma after surgery. Ann Surg Oncol. 2003;10:355-62.

16. Lencioni R, Llovet JM, Han G, Tak WY, Yang J, Guglielmi A, Paik SW, Reig M, Kim DY, Chau GY, Luca A, Del Arbol LR, Leberre MA, Niu W, Nicholson K, Meinhardt G, Bruix J. Sorafenib or placebo plus TACE with doxorubicin-eluting beads for intermediate stage HCC: the SPACE trial. J Hepatol. 2016;64:1090-8.

17. Meyer T, Fox R, Ma YT, Ross PJ, James MW, Sturgess R, Stubbs C, Stocken DD, Wall L, Watkinson A, Hacking N, Evans TRJ, Collins P, Hubner RA, Cunningham D, Primrose JN, Johnson PJ, Palmer DH. Sorafenib in combination with transarterial chemoembolisation in patients with unresectable hepatocellular carcinoma (TACE 2): a randomised placebo-controlled, double-blind, phase 3 trial. Lancet Gastroenterol Hepatol. 2017;2:565-75.

18. Kudo M, Han G, Finn RS, Poon RT, Blanc JF, Yan L, Yang J, Lu L, Tak WY, Yu X, Lee JH, Lin SM, Wu C, Tanwandee T, Shao G, Walters IB, Dela Cruz C, Poulart V, Wang JH. Brivanib as adjuvant therapy to transarterial chemoembolization in patients with hepatocellular carcinoma: a randomized phase III trial. Hepatology. 2014;60:1697-707.

19. Masatoshi Kudo, Kazuomi Ueshima, Masafumi Ikeda, Takuji Torimura, Nobukazu Tanabe, Hiroshi Aikata, Namiki Izumi, Takahiro Yamasaki, Shunsuke Nojiri, Keisuke Hino, Hidetaka Tsumura, Teiji Kuzuya, Norio Isoda, Kohichiroh Yasui, Kenichi Yoshimura, Takuji Okusaka, Junji Furuse, Norihiro Kokudo, Kiwamu Okita, Yasuaki Arai, For the TACTICS Trial Group. Randomized, open label, multicenter, phase II trial comparing transarterial chemoembolization (TACE) plus sorafenib with TACE alone in patients with hepatocellular carcinoma (HCC): TACTICS trial. Oral presented at: ASCO Gastrointestinal Cancer Symposium; January 2018; San Francisco, CA.

20. Kudo M, Cheng AL, Park JW, Park JH, Liang PC, Hidaka H, Izumi N, Heo J, Lee YJ, Sheen IS, Chiu CF, Arioka H, Morita $\mathrm{S}$, Arai Y. Orantinib versus placebo combined with transcatheter arterial chemoembolization in patients with unresectable hepatocellular carcinoma (ORIENTAL): a randomised, double-blind, placebo-controlled, multicentre, phase 3 study. Lancet Gastroenterol Hepatol. 2018;3:37-46.

21. Inaba Y, Kanai F, Aramaki T, Yamamoto T, Tanaka T, Yamakado K, Kaneko S, Kudo M, Imanaka K, Kora S, Nishida N, Kawai N, Seki H, Matsui O, Arioka H, Arai Y. A randomised phase II study of TSU-68 in patients with hepatocellular carcinoma treated by transarterial chemoembolisation. Eur J Cancer. 2013;49:2832-40.

22. Cheng AL, Amarapurkar D, Chao Y, Chen PJ, Geschwind JF, Goh KL, Han KH, Kudo M, Lee HC, Lee RC, Lesmana LA, Lim HY, Paik SW, Poon RT, Tan CK, Tanwandee T, Teng G, Park JW. Reevaluating transarterial chemoembolization for the treatment of hepatocellular carcinoma: consensus recommendations and review by an International Expert Panel. Liver Int. 2014;34:174-83.

23. European Association For The Study Of The Liver.; European Organisation For Research And Treatment Of Cancer. EASLEORTC clinical practice guidelines: management of hepatocellular carcinoma. J Hepatol 2012;56:908-43.

24. Yamakado K, Miyayama S, Hirota S, Mizunuma K, Nakamura K, Inaba Y, Maeda H, Matsuo K, Nishida N, Aramaki T, Anai H, Koura S, Oikawa S, Watanabe K, Yasumoto T, Furuichi K, Yamaguchi M. Subgrouping of intermediate-stage (BCLC stage 
B) hepatocellular carcinoma based on tumor number and size and Child-Pugh grade correlated with prognosis after transarterial chemoembolization. Jpn J Radiol. 2014;32:260-5.

25. Nakazawa T, Hidaka H, Shibuya A, Okuwaki Y, Tanaka Y, Takada J, Minamino T, Watanabe M, Kokubu S, Koizumi W. Overall survival in response to sorafenib versus radiotherapy in unresectable hepatocellular carcinoma with major portal vein tumor thrombosis: propensity score analysis. BMC Gastroenterol. 2014;14:84.

26. Zhang Y, Fan W, Wang Y, Lu L, Fu S, Yang J, Huang Y, Yao W, Li J. Sorafenib with and without transarterial chemoembolization for advanced hepatocellular carcinoma with main portal vein tumor thrombosis: a retrospective analysis. Oncologist. 2015;20:1417-24

27. Itoh Y, Okanoue T, Ohnishi N, Nishioji K, Sakamoto S, Nagao Y, Nakamura H, Kirishima T, Kashima K. Hepatic damage induced by transcatheter arterial chemoembolization elevates serum concentrations of macrophage-colony stimulating factor. Liver. 1999;19:97-103.

28. Hidaka H, Nakazawa T, Kaneko T, Minamino T, Takada J, Tanaka Y, Okuwaki Y, Watanabe M, Shibuya A, Koizumi W. Portal hemodynamic effects of sorafenib in patients with advanced hepatocellular carcinoma: a prospective cohort study. J Gastroenterol. 2012;47:1030-5.

Publisher's Note Springer Nature remains neutral with regard to jurisdictional claims in published maps and institutional affiliations.

\section{Affiliations}

Hisashi Hidaka ${ }^{1} \cdot$ Namiki Izumi $^{2} \cdot$ Takeshi Aramaki $^{3} \cdot$ Masafumi Ikeda $^{4} \cdot$ Yoshitaka Inaba $^{5} \cdot$ Kazuho Imanaka $^{6}$. Takuji Okusaka $^{7}$. Susumu Kanazawa ${ }^{8}$. Shuichi Kaneko ${ }^{9}$ Shinichi Kora ${ }^{10}$. Hiroya Saito ${ }^{11}$ • Junji Furuse ${ }^{12}$. Osamu Matsui ${ }^{13}$. Tatsuya Yamashita ${ }^{9} \cdot$ Osamu Yokosuka $^{14}$. Satoshi Morita ${ }^{15}$. Hitoshi Arioka ${ }^{16}$. Masatoshi Kudo ${ }^{17}$. Yasuaki Arai ${ }^{18}$

1 Department of Gastroenterology, Internal Medicine, Kitasato University School of Medicine, 1-15-1 Kitasato, Minami-ku, Sagamihara, Kanagawa 252-0380, Japan

2 Department of Gastroenterology and Hepatology, Musashino Red Cross Hospital, Tokyo, Japan

3 Division of Interventional Radiology, Shizuoka Cancer Center, Shizuoka, Japan

4 Department of Hepatobiliary and Pancreatic Oncology, National Cancer Center Hospital East, Chiba, Japan

5 Department of Diagnostic and Interventional Radiology, Aichi Cancer Center Hospital, Aichi, Japan

6 Department of Hepatobiliary and Pancreatic Oncology, Osaka International Cancer Institute, Osaka, Japan

7 Department of Hepatobiliary and Pancreatic Oncology, National Cancer Center Hospital, Tokyo, Japan

8 Department of Radiology, Okayama University Medical School, Okayama, Japan

9 Department of Gastroenterology, Kanazawa University Hospital, Ishikawa, Japan
10 Department of Radiology, Faculty of Medicine, Fukuoka University, Fukuoka, Japan

11 Department of Radiology, Asahikawa Kosei General Hospital, Hokkaido, Japan

12 Department of Medical Oncology, Kyorin University Faculty of Medicine, Tokyo, Japan

13 Department of Radiology, Kanazawa University Graduate School of Medical Science, Ishikawa, Japan

14 Department of Gastroenterology, Graduate School of Medicine, Chiba University, Chiba, Japan

15 Department of Biomedical Statistics and Bioinformatics, Kyoto University Graduate School of Medicine, Kyoto, Japan

16 Department of Medical Oncology, Yokohama Rosai Hospital, Kanagawa, Japan

17 Department of Gastroenterology and Hepatology, Kindai University Faculty of Medicine, Osaka, Japan

18 Department of Diagnostic Radiology, National Cancer Center Hospital, Tokyo, Japan 